\title{
Markers of acute kidney injury in patients with sepsis: the role of soluble thrombomodulin
}

Shinshu Katayama* (D), Shin Nunomiya, Kansuke Koyama, Masahiko Wada, Toshitaka Koinuma, Yuya Goto, Ken Tonai and Jun Shima

\begin{abstract}
Background: Endothelial activation and damage occur early during sepsis, with activated coagulopathy and playing a major role in the pathophysiology of sepsis-induced acute kidney injury (AKI). The aim of this study was to compare the various biomarkers of endothelial injury with the biomarkers of coagulation and inflammation and to determine a significant predictor of AKI in patients with sepsis.
\end{abstract}

Methods: We conducted a single-center, retrospective, observational study on patients with sepsis fulfilling the Third International Consensus Definitions for Sepsis and Septic Shock criteria admitted to an adult intensive care unit (ICU) at a university hospital from June 2011 to December 2016. Levels of 13 biomarkers were measured on ICU admission, including markers of endothelial injury (soluble thrombomodulin [sTM], E-selectin, protein C, and plasminogen activator inhibitor-1 [PAI-1]) and markers of coagulation derangement (platelet count, fibrin degradation product [FDP], prothrombin time [PT], fibrinogen, $\mathrm{a}_{2}$-plasminogen inhibitor [ $\left.\mathrm{a}_{2}-\mathrm{Pl}\right]$, antithrombin III [AT III], plasminogen, thrombinantithrombin complex, and plasmin- $a_{2}$-plasmin inhibitor complex). All patients with sepsis were reviewed, and the development of AKI was evaluated. Multivariate logistic regression analysis was performed to identify significant independent predictive factors for AKI.

Results: Of the 514 patients admitted with sepsis, 351 (68.3\%) developed AKI. Compared with the non-AKI group, all the endothelial biomarkers were significantly different in the AKI group (sTM [23.6 vs. $15.6 \mathrm{U} / \mathrm{ml}, P<0.0001]$, E-selectin [65.5 vs. $46.2 \mathrm{ng} / \mathrm{ml}, P=0.0497]$, PAl-1 [180.4 vs. $75.3 \mathrm{ng} / \mathrm{ml}, P=0.018]$, and protein C [45.9 vs. $58.7 \mathrm{ng} / \mathrm{ml}, P<0.0001]$ ). Biomarkers of coagulopathy and inflammation, platelet counts, FDP, PT, $\mathrm{a}_{2}-\mathrm{Pl}, \mathrm{AT} \mathrm{III}$, plasminogen, and C-reactive protein were significantly different between the two groups. Multivariable logistic regression analysis showed that STM was an independent predictive factor of AKI, with an AUROC of $0.758(P<0.0001)$.

Conclusions: Endothelial biomarkers were significantly changed in the sepsis patients with AKI. Particularly, sTM was an independent predictive biomarker for the development of AKI that outperformed other coagulation and inflammation biomarkers as well as organ function in patients with sepsis.

Keywords: Acute kidney injury, Endothelial injury, E-selectin, Sepsis, Soluble thrombomodulin, Plasminogen activator inhibitor-1, Protein C

\footnotetext{
*Correspondence: shinsyu_k@jichi.ac.jp

Division of Intensive Care, Department of Anesthesiology and Intensive Care

Medicine, Jichi Medical University School of Medicine, 3311-1, Yakushiji,

Shimotsuke, Tochigi 329-0498, Japan
} 


\section{Background}

Sepsis represents a life-threatening organ dysfunction caused by an aberrant or dysregulated host response to infection [1]. In particular, acute kidney injury (AKI) is one of the most frequent types of organ dysfunction that typically appears early in the course of sepsis. Nearly half of the patients develop AKI in the intensive care unit (ICU) setting, and the presence of AKI in these patients leads to an increase in mortality up to 30-50\% [2-4].

The high mortality rate associated with septic AKI may partially be explained by an incomplete understanding of its pathophysiology and a delay in diagnosis. Currently, the diagnosis of AKI is based on either an elevation of serum creatinine levels or the detection of oliguria [5]. These criteria, however, are not specific with respect to the etiology or pathophysiological mechanisms of AKI and have limited sensitivity and specificity for the early recognition of renal dysfunction. The introduction of new biomarkers that are related to the underlying pathogenesis of AKI may allow earlier diagnosis and better prognostication of the clinical course in patients with sepsis.

Endothelial injury is a key feature of sepsis, and the activation and damage of endothelial cells are closely linked with organ dysfunction [6, 7]. Previous studies highlighted systemic hypotension and ischemiareperfusion injury as the primary pathophysiology involved in septic AKI. Recently, however, it has been shown that septic AKI occurs in the setting of microvascular dysfunction, which is altered by endothelial injury [8-10]. In addition to their role in controlling vasoconstriction/vasodilation and vascular permeability, endothelial cells are essential for maintaining the balance between coagulation and anticoagulation, and they play a prominent role in all three major pathogenic pathways associated with coagulopathy in sepsis: increased tissue factor-mediated thrombin generation; dysfunction of antithrombin and the thrombomodulin-protein $\mathrm{C}$ anticoagulant system; and blocked fibrinolysis, which is induced by increased plasminogen activator inhibitor-1 (PAI-1) expression. During sepsis, exposure to inflammatory mediators and interaction with immune cells causes endothelial activation. The activated or injured endothelial cells exert enhanced procoagulatory activities, which contribute to the formation of a large number of microthrombi, leading to microcirculatory failure $[6,11]$. Therefore, markers that reflect endothelial injury and its associated coagulopathy could potentially predict the development of AKI and may play a role in promoting clinical research for underlying mechanisms.

To date, several biomarkers of endothelial injury have been evaluated regarding their association with AKI, such as endothelium-derived markers (e.g., thrombomodulin, PAI-1, E-selectin) and markers activated by endothelial cells (e.g., protein C) [12-17]. Most studies, however, were relatively small, conducted in the critical care setting, not focused on sepsis-induced AKI. Also, endothelial function is closely related to coagulopathy; however, there have been few studies in which this relationship has been evaluated. Finally, it remains unclear whether biomarkers of endothelial injury can predict the development of AKI in patients with sepsis.

We report our comparisons of various biomarkers of endothelial injury as predictors of AKI among 514 eligible patients with sepsis admitted to the ICU over a 5 -year study period. We also evaluated biomarkers of coagulopathy, parameters of inflammation and organ function, and parameters of endothelial injury to compare their discriminative power in AKI prediction.

\section{Methods}

\section{Study design and setting}

This was a single-center, retrospective, observational study conducted in a 14-bed general ICU of a university hospital (Tochigi, Japan) from June 2011 to December 2016. Laboratory tests, including those for measuring levels of biomarkers of endothelial injury and coagulopathy, were routinely performed at our institute and were partly used for published data [18]. Clinical decisions were made at the discretion of attending ICU physicians. The study protocol was approved by the institutional research ethics committee of Jichi Medical University Hospital. Informed consent was waived because of the retrospective nature of this study.

\section{Participants}

Patients were eligible for enrollment if they were aged 20 years or older and had sepsis at the time of ICU admission. Patients in whom levels of one of the endothelial biomarkers was measured were included in this study. Exclusion criteria included patients with endstage renal disease requiring dialysis, missing laboratory data on the day of ICU admission, or missing records of hourly urinary output or body weight, which are necessary for the diagnosis of AKI. The prothrombin time (PT) was excluded from the biomarkers of coagulopathy in patients for whom warfarin had been prescribed for any reason. Patient baseline data, including age, sex, body weight, site of infection, and premorbid creatinine level, were collected from electronic medical records. Underlying medical history was also obtained, including ischemic heart disease, chronic heart failure, chronic obstructive pulmonary disease, cerebrovascular accident, diabetes mellitus, or immunocompromised state. Immunocompromised patients were defined as individuals who had been prescribed any immunosuppressive agent. The Acute Physiology and Chronic Health Evaluation II (APACHE II) score [19] and Sequential Organ 
Failure Assessment (SOFA) score without the renal system parameter (nonrenal SOFA) [20] were used for assessment of illness severity. All patients were reviewed for the development of AKI during the first $48 \mathrm{~h}$ of their ICU stay and for the presence of septic shock, overt disseminated intravascular coagulation (DIC), requirement for mechanical ventilation, and mortality at 28 and 90 days.

\section{Definitions}

We defined AKI according to Kidney Disease: Improving Global Outcomes (KDIGO) criteria [5] using an increase in serum creatinine level $\geq 0.3 \mathrm{mg} / \mathrm{dl} \quad(\geq 26.5 \mu \mathrm{mol} / \mathrm{L})$ within $48 \mathrm{~h}$ or an increase in serum creatinine $\geq 1.5$ times of baseline within 7 days, which corresponds to stage 1 of the KDIGO classification. Chronic kidney disease (CKD) was defined as an estimated glomerular filtration rate $(\mathrm{eGFR})<60 \mathrm{ml} /$ minute $/ 1.73 \mathrm{~m}^{2}$ [21] using baseline creatinine. To define the baseline creatinine level, we used a stable value within 1 year before hospital admission as premorbid creatinine. If the baseline creatinine level was not available, the Modification of Diet in Renal Disease equation was used to assume a baseline eGFR of $75 \mathrm{ml} /$ minute $/ 1.73 \mathrm{~m}^{2}$ [22]. Sepsis and septic shock were defined according to the Third International Consensus Definitions for Sepsis and Septic Shock [1]. Overt DIC was defined according to the criteria of the International Society on Thrombosis and Haemostasis [23].

\section{Biomarker measurement and laboratory analysis}

Thirteen biomarkers of endothelial injury (soluble thrombomodulin [sTM], PAI-1, protein C, and Eselectin) and coagulation derangement (platelet count, fibrin degradation product [FDP], PT, fibrinogen, $\alpha_{2}-$ plasminogen inhibitor $\left[\alpha_{2}-\mathrm{PI}\right]$, antithrombin III [AT III], plasminogen, thrombin-antithrombin complex [TAT], plasmin- $\alpha_{2}$-plasmin inhibitor complex [PIC]) were measured on ICU admission. sTM concentration was determined using the STACIA chemiluminescence enzyme immunoassay with reagent (LSI Medience, Tokyo, Japan). The levels of PAI-1 and E-selectin were measured using tissue plasminogen activator inhibitor and sES latex photometric immunoassays, respectively (Mitsubishi Chemical Medience, Tokyo, Japan). Berichrom ${ }^{\bullet}$ assays (Siemens Healthcare Diagnostics, Tokyo, Japan) were used to determine the activities of protein C, AT III, plasminogen, and $\alpha_{2}$-PI. TAT and PIC F enzyme immunoassays (Sysmex, Hyogo, Japan) were used for measurement of TAT and PIC levels, respectively. Blood coagulation, including those for platelet count, PT, FDP, and fibrinogen, was assayed using the CS-2100i automatic coagulation analyzer (Sysmex). We also measured the following inflammatory and biochemistry parameters on the day of ICU admission: C-reactive protein (CRP), white blood cell count (WBC), blood urea nitrogen (BUN), serum creatinine, albumin, total bilirubin, lactate, brain natriuretic peptide (BNP), and serum cystatin C.

\section{Statistical analysis}

Categorical variables were compared between the two groups using the chi-square test. To determine factors relevant for predicting AKI, we conducted multivariate logistic regression analysis with AKI as the dependent variable and evaluated unadjusted and adjusted ORs. In this analysis, we used model 1 (endothelial biomarkers [sTM and protein C], all coagulation biomarkers including overt DIC, and inflammation parameters [WBC and CRP]) and model 2 (endothelial biomarkers, all coagulation biomarkers including overt DIC, inflammation parameters, organ function [APACHE II, baseline creatinine, BNP, nonrenal SOFA, albumin, total bilirubin, hemoglobin, and presence of septic shock], age, and presence of hypertension) to calculate the adjusted OR. A forward stepwise elimination process was used to remove insignificant variables by each model. The ability of biomarkers to predict AKI was assessed using the AUROC [24]. All analyses were performed using JMP 13 software (SAS Institute, Cary, NC, USA). Data are presented as medians and IQRs (25th-75th percentiles) or percentages. $P$ values $<0.05$ were considered significant.

\section{Results \\ Enrollment and baseline characteristics}

There were 5764 patients considered for participation during the study period. Among these, 651 patients presented with sepsis. Among the patients who were excluded, 50 had received dialysis because of end-stage renal failure, 5 had omission of hourly urinary output or body weight, and 82 had omission of laboratory data on the day of ICU admission. In total, 514 patients were included and divided into the AKI $(n=351)$ and nonAKI $(n=163)$ groups (Table 1$)$. In the AKI group compared with the non-AKI group, age was significantly older (71 vs. 65 years, $P=0.003$ ); APACHE II score was significantly higher (26 vs. $19, P<0.0001$ ); and CKD was significantly more common ( $31.1 \%$ vs. $21.5 \%, P=0.032)$. Mechanical ventilation was significantly higher $(85.8 \%$ vs. $76.7 \%, P=0.011)$ in the AKI group. The 28 -day $(15.6 \%$ vs. $3.1 \%, P<0.0001)$ and 90 -day $(24.5 \%$ vs. $8.4 \%, P<0.0001)$ mortality rates were also significantly higher in the AKI group than in the non-AKI group.

\section{Laboratory tests and endothelial biomarkers in AKI and non-AKI groups}

Table 2 shows the measured variables between the two groups. Among the endothelial biomarkers, sTM (23.6 vs. $15.6 \mathrm{U} / \mathrm{ml}, P<0.0001)$ and E-selectin $(65.5$ vs. $46.2 \mathrm{ng} / \mathrm{ml}, P=0.0497)$ levels were both significantly 
Table 1 Characteristics of the study population

\begin{tabular}{|c|c|c|c|c|}
\hline & All $(n=514)$ & AKI $(n=163)$ & Non-AKI $(n=351)$ & $P$ value \\
\hline Age, years & 69 (59-78) & $65(56-74)$ & $71(61-79)$ & 0.0003 \\
\hline BW, kg & $56.7(48-66)$ & $55(46-64)$ & $57(49-67)$ & 0.336 \\
\hline Height, cm & $159(151-166)$ & $159(152-166)$ & $160(150-166)$ & 0.823 \\
\hline Male sex & $53.9 \%$ & $53.4 \%$ & $54.1 \%$ & 0.873 \\
\hline APACHE ॥ & $24(18-30)$ & $19(15-25)$ & $26(20-31)$ & $<0.0001$ \\
\hline CKD & $28.0 \%$ & $21.5 \%$ & $31.1 \%$ & 0.032 \\
\hline Premorbid creatinine, $\mu \mathrm{mol} / \mathrm{L}$ & $66.3(53.0-84.9)$ & $61.9(47.7-80.0)$ & $70.3(53.9-89.5)$ & 0.007 \\
\hline Premorbid creatinine, mg/dl & $0.75(0.60-0.96)$ & $0.70(0.54-0.91)$ & $0.80(0.0 .61-1.01)$ & 0.007 \\
\hline Baseline creatinine, $\mu \mathrm{mol} / \mathrm{L}$ & $54.8(50.9-71.6)$ & $55.1(51.3-65.4)$ & $54.8(50.8-72.5)$ & 0.011 \\
\hline Baseline creatinine, mg/dl & $0.62(0.58-0.81)$ & $0.62(0.58-0.74)$ & $0.62(0.57-0.82)$ & 0.011 \\
\hline Hypertension & $48.8 \%$ & $39.3 \%$ & $53.3 \%$ & 0.003 \\
\hline $\mathrm{IHD}$ & $8.8 \%$ & $5.5 \%$ & $10.3 \%$ & 0.077 \\
\hline $\mathrm{CHF}$ & $8.8 \%$ & $4.9 \%$ & $10.5 \%$ & 0.036 \\
\hline COPD & $5.1 \%$ & $7.4 \%$ & $4.0 \%$ & 0.104 \\
\hline CVA & $11.1 \%$ & $8.6 \%$ & $12.3 \%$ & 0.219 \\
\hline $\mathrm{DM}$ & $25.7 \%$ & $21.5 \%$ & $27.6 \%$ & 0.137 \\
\hline Immunocompromised & $29.2 \%$ & $27.6 \%$ & $29.9 \%$ & 0.592 \\
\hline Infection site & & & & 0.013 \\
\hline Intracranial & $1.2 \%$ & $2.5 \%$ & $0.6 \%$ & \\
\hline Head and neck & $5.5 \%$ & $9.2 \%$ & $3.7 \%$ & \\
\hline Thoracic & $22.4 \%$ & $22.1 \%$ & $22.5 \%$ & \\
\hline Abdominal & $50.8 \%$ & $53.4 \%$ & $49.6 \%$ & \\
\hline Soft tissue & $6.0 \%$ & $4.9 \%$ & $6.6 \%$ & \\
\hline CR-BSI & $1.0 \%$ & $0.0 \%$ & $1.4 \%$ & \\
\hline UTI & $5.3 \%$ & $3.7 \%$ & $6.0 \%$ & \\
\hline Others & $8.0 \%$ & $4.3 \%$ & $10.3 \%$ & \\
\hline Mechanical ventilation & $82.9 \%$ & $76.7 \%$ & $85.8 \%$ & 0.011 \\
\hline 28-day mortality & $11.7 \%$ & $3.1 \%$ & $15.6 \%$ & $<0.0001$ \\
\hline 90-day mortality & $19.3 \%$ & $8.4 \%$ & $24.5 \%$ & $<0.0001$ \\
\hline
\end{tabular}

Abbreviations: AKI Acute kidney injury, APACHE II Acute Physiology and Chronic Health Evaluation II score, BMI Body mass index, BW Body weight, CHD Chronic heart disease, $C K D$ Chronic kidney disease, COPD Chronic obstructive pulmonary disease, $C R$-BSI Catheter-related bloodstream infection, CVA Cerebrovascular accident, DM Diabetes mellitus, IHD Ischemic heart disease, UTI Urinary tract infection

higher in patients with AKI than in those without AKI. Also, protein $C$ activity $(45.9 \%$ vs. $58.7 \%, P<0.0001)$ was significantly lower, and the level of PAI-1 (180.4 vs. $75.3 \mathrm{ng} / \mathrm{ml}, P=0.018$ ) was significantly higher, in the AKI group. As for parameters of inflammation and organ function, the following were all significantly different in the AKI group compared with in the non-AKI group: CRP, BUN, serum creatinine, cystatin $\mathrm{C}, \mathrm{BNP}$, albumin, hemoglobin, lactate, the proportion of patients with septic shock, nonrenal SOFA score, and number of overt DIC cases. As for the coagulation biomarkers, platelet count, PT, FDP, AT III, plasminogen, and $\alpha_{2}$-PI were significantly different for patients in the AKI group compared with in the non-AKI group. In addition, each variable for biomarkers of endothelial injury and coagulopathy was analyzed using AUROC (Table 3). The AUROC values for sTM, E-selectin, protein $\mathrm{C}$, and PAI-1 as predictive factors were 0.758 (0.677-0.825), 0.629 (0.492-0.748), 0.634 (0.581-0.685), and $0.669(0.566-0.758)$, respectively.

\section{Multivariate analysis to identify factors predictive of AKI}

To identify the predictable factors of AKI, we performed multivariate logistic regression analysis using model 1 and model 2. In both models, sTM was an independent significant predictor of AKI. The nonadjusted OR of sTM was $1.11(1.06-1.17, P<0.003)$; the adjusted OR of model 1 was $1.10(1.04-1.16, P=0.001)$ and that of model 2 was $1.09(1.04-1.16, P=0.004)$ (Table 4$)$. 
Table 2 Laboratory tests and endothelial biomarkers

\begin{tabular}{|c|c|c|c|c|}
\hline & All $(n=514)$ & Non-AKI $(n=163)$ & $\operatorname{AKI}(n=351)$ & $P$ value \\
\hline \multicolumn{5}{|l|}{ Laboratory tests } \\
\hline $\mathrm{WBC}, 10^{9} / \mathrm{L}$ & $9.9(4.2-15.0)$ & $9.5(5.0-14.2)$ & $10.1(3.7-15.2)$ & 0.255 \\
\hline $\mathrm{Hb}, \mathrm{g} / \mathrm{L}$ & $105(89-121)$ & $107(93-122)$ & $104(86-120)$ & 0.023 \\
\hline$C R P, m g / L$ & $128(62-225)$ & $105(41-186)$ & $137(70-242)$ & 0.002 \\
\hline Alb, g/L & $24(20-28)$ & $24(21-28)$ & $23(19-28)$ & 0.013 \\
\hline T-Bil, $\mu \mathrm{mol} / \mathrm{L}$ & $15.2(10.8-24.8)$ & $15.6(11.1-23.8)$ & $15.0(10.6-25.8)$ & 0.395 \\
\hline Lactate, $\mathrm{mmol} / \mathrm{L}$ & $2.2(1.4-3.7)$ & $1.9(1.2-2.7)$ & $2.5(1.5-4.6)$ & $<0.0001$ \\
\hline $\mathrm{BNP}, \mathrm{ng} / \mathrm{L}$ & $136.7(50.6-395.1)$ & $70.2(29.8-204.8)$ & $175.0(68.1-522.7)$ & 0.0003 \\
\hline \multicolumn{5}{|l|}{ Renal parameters } \\
\hline $\mathrm{BUN}, \mathrm{mmol} / \mathrm{L}$ & $8.9(5.4-14.6)$ & $5.4(3.9-7.9)$ & $11.4(7.5-16.6)$ & $<0.0001$ \\
\hline Creatinine, $\mu \mathrm{mol} / \mathrm{L}$ & $89.7(61.0-162.9)$ & $57.5(47.7-69.0)$ & $127.3(84.9-206.9)$ & $<0.0001$ \\
\hline Creatinine, mg/dl & $1.02(0.69-1.84)$ & $0.65(0.54-0.78)$ & $1.44(0.96-2.34)$ & $<0.0001$ \\
\hline Cystatin C, mg/L & $1.29(0.91-2.02)$ & $0.86(0.72-1.03)$ & $1.65(1.14-2.29)$ & $<0.0001$ \\
\hline \multicolumn{5}{|l|}{ Coagulation biomarkers } \\
\hline Platelets, $10^{9} / \mathrm{L}$ & $144(91-207)$ & $167(127-238)$ & $131(78-193)$ & $<0.0001$ \\
\hline $\mathrm{FDP}, \mathrm{mg} / \mathrm{L}$ & $16.7(10.0-27.6)$ & $13.6(8.9-21.7)$ & $18.9(10.9-30.5)$ & 0.049 \\
\hline PT, \% & $56.4(44.3-70.3)$ & $63.3(50.3-75.8)$ & $52.4(42.5-67.6)$ & $<0.0001$ \\
\hline $\mathrm{Fib}, \mathrm{mg} / \mathrm{dl}$ & $343(242-487)$ & $373(261-528)$ & $333(234-471)$ & 0.076 \\
\hline $\mathrm{a}_{2}-\mathrm{PI}, \%$ & $75.2(57.9-97.2)$ & $82.0(63.3-102.0)$ & 73.6 (56.9-94.0) & 0.029 \\
\hline AT III, \% & $53.7(41.9-69.0)$ & $60.4(45.5-76.3)$ & $51.5(39.3-63.0)$ & $<0.0001$ \\
\hline Plasminogen, \% & $61.4(46.2-80.3)$ & $66.9(52.9-88.8)$ & $59.7(43.6-77.6)$ & 0.0002 \\
\hline TAT, ng/ml & $11.1(6.2-20.0)$ & $9.2(5.4-16.7)$ & $12.4(6.7-21.5)$ & 0.088 \\
\hline $\mathrm{PIC}, \mu \mathrm{g} / \mathrm{ml}$ & $1.3(0.8-2.1)$ & $1.2(0.8-1.9)$ & $1.3(0.8-2.2)$ & 0.613 \\
\hline \multicolumn{5}{|l|}{ Endothelial activation } \\
\hline Protein C, \% & $49.6(36.3-66.4)$ & $58.7(44.2-74.0)$ & $45.9(33.6-63.6)$ & $<0.0001$ \\
\hline sTM, U/ml & $21.1(15.4-31.5)$ & $15.6(12.6-20.5)$ & $23.6(17.0-38.8)$ & $<0.0001$ \\
\hline E-selectin, ng/ml & $55.2(36.2-98.2)$ & $46.2(32.3-67.5)$ & $65.5(40.2-131.0)$ & 0.0497 \\
\hline $\mathrm{PAl}-1, \mathrm{ng} / \mathrm{ml}$ & 116.3 (53.8-290.8) & $75.3(40.3-150.7)$ & $180.4(68.0-519.1)$ & 0.018 \\
\hline \multicolumn{5}{|l|}{ Other definitions } \\
\hline No renal SOFA & $6(4-9)$ & $5(3-7)$ & $7(5-9)$ & $<0.0001$ \\
\hline Overt DIC & $24.5 \%$ & $10.1 \%$ & $31.6 \%$ & $<0.0001$ \\
\hline Septic shock & $45.7 \%$ & $29.5 \%$ & $53.3 \%$ & $<0.0001$ \\
\hline
\end{tabular}

Abbreviations: AKI Acute kidney injury, Alb Albumin, AT III Antithrombin III, BNP Brain natriuretic peptide, BUN Blood urea nitrogen, CRP C-reactive protein, $D I C$ Disseminated intravascular coagulation, FDP Fibrin degradation product, $H b$ Hemoglobin, PAl-1 Plasminogen activator inhibitor-1, $a_{2}-P l a_{2}-P l a s m i n o g e n$ inhibitor, PIC Plasmin- $a_{2}$-plasmin inhibitor complex, PT Prothrombin time, SOFA Sequential Organ Failure Assessment, sTM Soluble thrombomodulin,

TAT Thrombin-antithrombin complex, T-Bil Total bilirubin, WBC White blood cell

Relationship of sTM and CKD at time of ICU admission Researchers in previous studies have reported that sTM was elevated in patients with CKD compared with healthy subjects $[24,25]$. Therefore, we analyzed whether the sTM level was different according to the presence or absence of CKD (premorbid eGFR $<60 \mathrm{ml} /$ minute $/ 1.73 \mathrm{~m}^{2}$ ). In the non-AKI group, there was a significant difference in sTM levels between patients who presented with vs. without CKD (20.4 vs. $13.6 \mathrm{U} / \mathrm{ml}, P=0.049$ ). Furthermore, in both groups with and without CKD, patients who presented with AKI had significantly higher levels of sTM than those without AKI (31.4 vs. $20.4 \mathrm{U} / \mathrm{ml}, P=0.013 ; 22.9$ vs. 13.6 $\mathrm{U} / \mathrm{ml}, P=0.001$, respectively), suggesting that the elevated sTM was related more to the development of AKI than to baseline renal function (Fig. 1).

\section{Discussion}

In this study, the AKI group presented not only endothelial injury but also activation of the coagulation system and the development of organ dysfunction. 
Table 3 AUROCs for predictors of acute kidney injury in sepsis

\begin{tabular}{ll}
\hline & AUROC (95\% CI) \\
\hline Coagulation biomarkers & \\
Platelet & $0.627(0.576-0.675)$ \\
FDP & $0.614(0.562-0.663)$ \\
PT & $0.629(0.576-0.680)$ \\
Fib & $0.549(0.494-0.602)$ \\
a $_{2}$-PI & $0.564(0.509-0.618)$ \\
AT III & $0.618(0.564-0.670)$ \\
Plasminogen & $0.600(0.545-0.652)$ \\
TAT & $0.591(0.538-0.642)$ \\
PIC & $0.527(0.475-0.579)$ \\
Overt DIC & $0.607(0.572-0.641)$ \\
Endothelial activation & \\
Protein C & $0.634(0.581-0.685)$ \\
STM & $0.758(0.677-0.825)$ \\
E-selectin & $0.629(0.492-0.748)$ \\
PAI-1 & $0.669(0.566-0.758)$ \\
\hline
\end{tabular}

Abbreviations: AKI Acute kidney injury, AT III Antithrombin III, DIC Disseminated intravascular coagulation, FDP Fibrin degradation product, PAl-1 Plasminogen activator inhibitor-1, $a_{2} \mathrm{Pl}_{2} \mathrm{a}_{2}$-Plasminogen inhibitor, PIC Plasmin- $\mathrm{a}_{2}$-plasmin inhibitor complex, PT Prothrombin time, sTM Soluble thrombomodulin, TAT Thrombin-antithrombin complex
However, multivariate logistic regression analysis showed that only sTM was an independent significant factor compared with several indices of coagulation activation, inflammation, and organ dysfunction. During the clinical course of sepsis, endothelial injury may occur initially, and then subsequent activation of coagulation leads to organ dysfunction [10]. On the basis of results of multivariable logistic regression analysis, we speculate that endothelial injury occurs as a result of inflammation in sepsis, which in turn results in AKI.

Thrombomodulin (TM) is a thrombin receptor expressed on the surface of endothelial cells. Once thrombin binds with endothelial TM, protein $C$ is activated, and STM is released into the bloodstream, which inactivates the procoagulant function of thrombin. There is some evidence that increased sTM levels are indicative of endothelial injury, with correlations to DIC, multiple organ failure (MOF), and mortality [7, 26, 27]. However, only three studies have been focused on the relationship between AKI and sTM. In a multicenter, prospective, observational study, sTM and protein C were shown to be predictable markers of AKI in critical care settings [16], whereas in another study, sTM and angiopoietin-2 were shown to play important roles in the development of AKI in patients with acute myocardial infarction [17]. Investigators in another study reported that the high levels of sTM were associated with reduction in the chance of recovery prior to renal function in patients

Table 4 Logistic regression analysis for determining predictors of acute kidney injury in sepsis

\begin{tabular}{|c|c|c|c|c|c|c|}
\hline & OR (95\% Cl) (unadjusted) & $P$-value & OR (95\% CI) (adjusted: model 1) & $P$ value & OR (95\% CI) (adjusted: model 2) & $P$ value \\
\hline \multicolumn{7}{|c|}{ Coagulation biomarkers } \\
\hline Platelet & $1.00(0.99-1.00)$ & $<0.0001$ & - & - & - & - \\
\hline FDP & $1.01(1.00-1.02)$ & 0.022 & - & - & - & - \\
\hline PT & $0.98(0.97-0.99)$ & $<0.0001$ & - & - & - & - \\
\hline $\mathrm{Fib}$ & $1.00(1.00-1.00)$ & 0.077 & - & - & - & - \\
\hline$a_{2}-P I$ & $0.99(0.99-1.00)$ & 0.029 & - & - & - & - \\
\hline AT III & $0.98(0.97-0.99)$ & $<0.0001$ & - & - & - & - \\
\hline Plasminogen & $0.99(0.98-0.99)$ & 0.0003 & - & - & - & - \\
\hline TAT & $1.02(1.01-1.04)$ & 0.0004 & - & - & - & - \\
\hline PIC & $1.02(0.95-1.11)$ & 0.603 & - & - & - & - \\
\hline Overt DIC & $4.10(2.39-7.47)$ & $<0.0001$ & - & - & - & - \\
\hline \multicolumn{7}{|c|}{ Endothelial activation } \\
\hline Protein C & $0.98(0.98-0.99)$ & $<0.0001$ & - & - & - & - \\
\hline STM & $1.11(1.06-1.17)$ & $<0.0001$ & $1.10(1.04-1.16)$ & 0.001 & $1.09(1.03-1.16)$ & 0.004 \\
\hline E-selectin & $1.01(1.00-1.02)$ & 0.029 & - & - & - & - \\
\hline PAl-1 & $1.00(1.00-1.00)$ & 0.007 & - & - & - & - \\
\hline
\end{tabular}

Abbreviations: AKI Acute kidney injury, AT III Antithrombin III, DIC Disseminated intravascular coagulation, FDP Fibrin degradation product, PAI-1 Plasminogen activator inhibitor-1, $a_{2} P I a_{2}$-Plasminogen inhibitor, PIC Plasmin- $a_{2}$-plasmin inhibitor complex, PT Prothrombin time, sTM Soluble thrombomodulin, TAT Thrombin-antithrombin complex

Model 1 included endothelial biomarkers, all coagulation biomarkers including overt DIC, and inflammation factors. Model 2 included endothelial biomarkers, all coagulation factors including overt DIC, inflammation factors, organ functions, and age and hypertension 


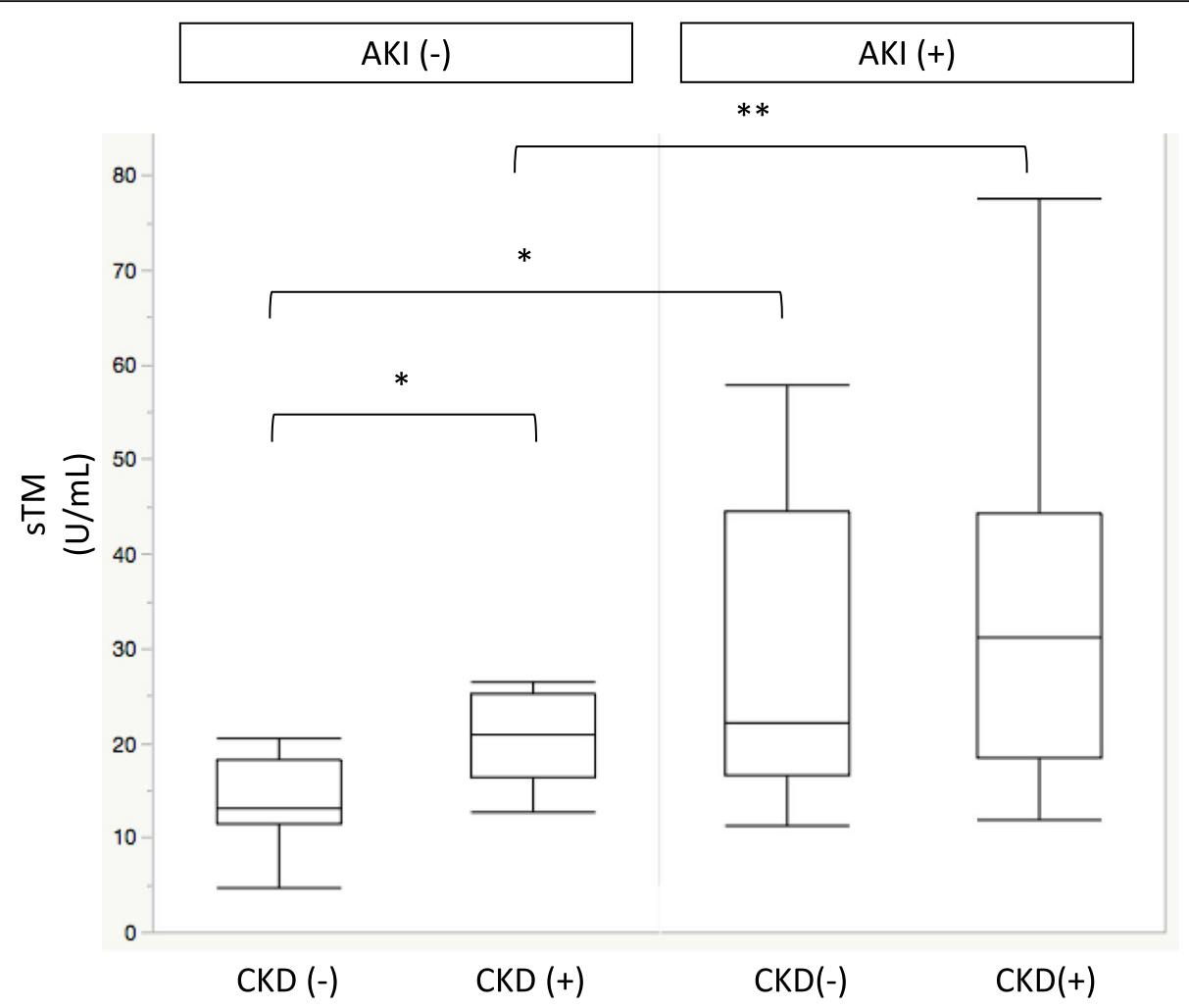

Fig. 1 Soluble thrombomodulin (STM) levels in patients with acute kidney injury (AKI) or without AKI differentiated by the presence of chronic kidney disease (CKD). * $P<0.05$, ** $P<0.01$

with AKI [28]. However, neither of these studies was focused specifically on patients with sepsis and evaluated the comparison between endothelial activity and coagulopathy. In this study, we used two models of multivariate logistic regression analysis to evaluate the relationship between endothelial function, coagulopathy, and inflammation because these parameters are related to each other. The results indicated that elevated sTM, as a marker of endothelial injury, was a strong independent predictive factor for AKI, regardless of coagulopathy, inflammation, and organ function.

In previous studies, researchers have reported that elevated sTM and protein $C$ levels are strong predictors of AKI in critically ill patients [16]. In this study, protein C was significantly decreased in AKI; however, protein $\mathrm{C}$ was not an independent factor in multivariable analysis. This may be partly because our study population comprised patients with sepsis, which is different from previous studies. Coagulation disorders frequently occur in sepsis and are associated with inflammation, which may account for the lack of significant differences in protein $\mathrm{C}$ levels between the groups. In this study, we evaluated coagulation function in more detail; however, even when these were factored into the multivariate analysis, only sTM, and not protein $C$, remained independently predictive of AKI. These results indicate that protein $\mathrm{C}$ is more related to coagulation behavior; it may not be a good predictor for AKI.

Some studies have indicated that STM levels increase in patients with CKD because sTM is excreted by the kidney [25, 29]. Kazama et al. reached this conclusion on the basis of the relationship between decreased urinary TM excretion and increased plasma levels of sTM in patients with CKD [29]. However, no data were available for either creatinine levels or baseline characteristics in their study. In contrast, Lin et al. showed that there was no relationship between sTM and creatinine clearance in patients with septic DIC and/or MOF [7]. Although we did not measure urinary TM in this study, there was a relationship between sTM and CKD in the non-AKI group. However, there was a significant difference in sTM levels in the AKI group, regardless of the presence or absence of CKD. Besides, compared with sTM, the baseline creatinine level was not an independent factor predictive of AKI. In this study, high levels of sTM suggest the predomination of endothelial injury rather than decreases in sTM clearance in patients with sepsis.

In two other studies, researchers found that levels of Eselectin significantly increased in cases of AKI in sepsis, but they measured only the levels of intracellular adhesion molecule-1 (ICAM-1), vascular cell adhesion molecule-1 (VCAM-1), and E-selectin as markers of endothelial injury 
and did not measure coagulopathy $[12,13]$. Therefore, it is unclear whether AKI or coagulation disorders were more predominant, because the presence of coagulation disorders was not verified in these studies. In our study, Eselectin presented with a significantly increased level in the AKI group, but it was not an independent factor with a low AUROC of 0.629. Therefore, we considered it to be insufficient for use as a predictive marker for AKI in sepsis if used as a single parameter.

This study has several strengths. One strength is that this is the first study, to our knowledge, including assessment of sTM as a parameter to evaluate endothelial injury during AKI in sepsis. Another strength is that we provide a detailed examination of coagulation parameters, as well as multivariate analysis and AUROC of the various coagulation parameters, including those of the criteria for overt DIC, which showed that there was an independent relationship between endothelial injury and AKI.

However, our study also has several limitations. First, this study was a single-center, retrospective, observational study. Second, we evaluated four parameters of endothelial injury, but we did not measure other parameters, such as ICAM-1 or VCAM-1. Further studies are needed to evaluate ideal predictive endothelial biomarkers for AKI. Third, data were not available for some biological markers during admission to the ICU. However, this study included a relatively large numbers of patients, which makes these results reliable. Fourth, there was a possibility that AKI had already occurred before admission to the ICU. Therefore, timing of AKI was not always matched for all patients. Further studies are needed to confirm the relationship between endothelial biomarkers and timing of AKI. Finally, we did not evaluate other biomarkers specifically related to AKI, such as urinary neutrophil gelatinase-associated lipocalin [30] and kidney injury molecule-1 [31]. AKI was not only caused by endothelial injury but also confounded by factors such as ischemia or drug toxicity. Therefore, such biomarkers may be superior for the early detection of AKI. However, it is important to evaluate the pathophysiology of AKI in sepsis; it may be associated with any potential clinical intervention for each cause. In this regard, the combination of several biomarkers and definition of AKI may be ideal for evaluating the cause of AKI in sepsis.

\section{Conclusions}

We show that STM is an independent predictor of AKI in sepsis that is superior to other coagulation and inflammation biomarkers as well as organ function. However, further studies are needed to clarify the relationship between biomarkers of endothelial injury and the development of AKI in sepsis. With time, it is hoped that this will facilitate early recognition of endothelial injury and early treatment.

\section{Abbreviations}

AKl: Acute kidney injury; Alb: Albumin; APACHE Il: Acute Physiology and Chronic Health Evaluation II; AT III: Antithrombin III; BMI: Body mass index; BNP: Brain natriuretic peptide; BUN: Blood urea nitrogen; BW: Body weight; CHD: Chronic heart disease; CKD: Chronic kidney disease; COPD: Chronic obstructive pulmonary disease; CR-BSI: Catheter-related bloodstream infection; CRP: C-reactive protein; CVA: Cerebrovascular accident; DIC: Disseminated intravascular coagulation; DM: Diabetes mellitus; eGFR: Estimated glomerular filtration rate; FDP: Fibrin degradation product; Hb: Hemoglobin; ICAM-1: Intracellular adhesion molecule-1; ICU: Intensive care unit; IHD: Ischemic heart disease; KDIGO: Kidney Disease: Improving Global Outcomes; MOF: Multiple organ failure; PAI-1: Plasminogen activator inhibitor-1; $a_{2}-P I$ : $a_{2}$-Plasminogen inhibitor; PIC: Plasmin- $a_{2}$-plasmin inhibitor complex; PT: Prothrombin time; SOFA: Sequential Organ Failure Assessment; sTM: Soluble thrombomodulin; TAT: Thrombin-antithrombin complex; T-bil: Total bilirubin; TM: Thrombomodulin; UTI: Urinary tract infection; VCAM-1: Vascular cell adhesion molecule-1; WBC: White blood cell count

\section{Acknowledgements}

We appreciate the assistance from the nursing staff of the intensive care unit at Jichi Medical University Hospital, Tochigi, Japan.

\section{Funding}

The study was conducted with the help of internal departmental funds.

\section{Availability of data and materials}

The dataset generated and/or analyzed during the present study is not publicly available, owing to patient-related confidentiality, but it is available from the corresponding author on reasonable request.

\section{Authors' contributions}

SK collected the data, interpreted the statistical results, and wrote the first draft of the manuscript. SN and KK contributed to the design of the protocol and revised the manuscript. YG collected the data and revised the manuscript. MW, TK, KT, and JS revised the manuscript. All authors are accountable for all aspects of the work, and all authors read and approved the final manuscript.

\section{Ethics approval and consent to participate}

This study was approved by the institutional review board of Jichi Medical University (Tochigi, Japan) (16-116). Informed consent was waived on the basis of the study's retrospective, observational design and preservation of confidentiality of personal information.

\section{Consent for publication}

Not applicable.

\section{Competing interests}

The authors declare that they have no competing interests.

\section{Publisher's Note}

Springer Nature remains neutral with regard to jurisdictional claims in published maps and institutional affiliations.

Received: 30 May 2017 Accepted: 8 August 2017

Published online: 25 August 2017

\section{References}

1. Singer M, Deutschman CS, Seymour CW, Shankar-Hari M, Annane D, Bauer $M$, et al. The Third International Consensus Definitions for Sepsis and Septic Shock (Sepsis-3). JAMA. 2016;315:801-10.

2. Bagshaw SM, Uchino S, Bellomo R, Morimatsu H, Morgera S, Schetz M, et al. Septic acute kidney injury in critically ill patients: clinical characteristics and outcomes. Clin J Am Soc Nephrol. 2007;2:431-9.

3. Kolhe NV, Stevens PE, Crowe AV, Lipkin GW, Harrison DA. Case mix, outcome and activity for patients with severe acute kidney injury during the first 24 hours after admission to an adult, general critical care unit: application of predictive models from a secondary analysis of the ICNARC Case Mix Programme database. Crit Care. 2008;12 Suppl 1:S2.

4. Bagshaw SM, George C, Bellomo R. Early acute kidney injury and sepsis: a multicentre evaluation. Crit Care. 2008;12:R47. 
5. Kellum JA, Lameire N. Diagnosis, evaluation, and management of acute kidney injury: a KDIGO summary (part 1). Crit Care. 2013;17:204.

6. Shapiro NI, Schuetz P, Yano K, Sorasaki M, Parikh SM, Jones AE, et al. The association of endothelial cell signaling, severity of illness, and organ dysfunction in sepsis. Crit Care. 2010;14:R182.

7. Lin SM, Wang YM, Lin HC, Lee KY, Huang CD, Liu CY, et al. Serum thrombomodulin level relates to the clinical course of disseminated intravascular coagulation, multiorgan dysfunction syndrome, and mortality in patients with sepsis. Crit Care Med. 2008;36:683-9.

8. Schrier RW, Wang W. Acute renal failure and sepsis. N Engl J Med. 2004;351:159-69.

9. Umbro I, Gentile G, Tinti F, Muiesan P, Mitterhofer AP. Recent advances in pathophysiology and biomarkers of sepsis-induced acute kidney injury. J Infect. 2016;72:131-42.

10. Doi K. Role of kidney injury in sepsis. J Intensive Care. 2016;4:17.

11. Reinhart K, Bayer O, Brunkhorst F, Meisner M. Markers of endothelial damage in organ dysfunction and sepsis. Crit Care Med. 2002;30(5 Suppl):S302-12.

12. Su CM, Cheng HH, Hung CW, Hsiao SY, Tsai NW, Chang WN, et al. The value of serial serum cell adhesion molecules in predicting acute kidney injury after severe sepsis in adults. Clin Chim Acta. 2016;457:86-91.

13. Powell TC, Powell SL, Allen BK, Griffin RL, Warnock DG, Wang HE. Association of inflammatory and endothelial cell activation biomarkers with acute kidney injury after sepsis. Springerplus. 2014;3:207.

14. Kümpers $P$, Hafer $C$, David S, Hecker H, Lukasz A, Fliser D, et al. Angiopoietin-2 in patients requiring renal replacement therapy in the ICU: relation to acute kidney injury, multiple organ dysfunction syndrome and outcome. Intensive Care Med. 2010;36:462-70.

15. Sadik NA, Mohamed WA, Ahmed MI. The association of receptor of advanced glycated end products and inflammatory mediators contributes to endothelial dysfunction in a prospective study of acute kidney injury patients with sepsis. Mol Cell Biochem. 2012;359:73-81.

16. Bouchard J, Malhotra R, Shah S, Kao YT, Vaida F, Gupta A, et al. Levels of protein $\mathrm{C}$ and soluble thrombomodulin in critically ill patients with acute kidney injury: a multicenter prospective observational study. PLoS One. 2015;10, e0120770.

17. Liu KL, Lee KT, Chang $\mathrm{CH}$, Chen YC, Lin SM, Chu PH. Elevated plasma thrombomodulin and angiopoietin-2 predict the development of acute kidney injury in patients with acute myocardial infarction. Crit Care. 2014;18:R100.

18. Koyama K, Madoiwa S, Nunomiya S, Koinuma T, Wada M, Sakata A, et al. Combination of thrombin-antithrombin complex, plasminogen activator inhibitor-1, and protein C activity for early identification of severe coagulopathy in initial phase of sepsis: a prospective observational study. Crit Care. 2014;18:R13.

19. Knaus WA, Draper EA, Wagner DP, Zimmerman JE. APACHE II: a severity of disease classification system. Crit Care Med. 1985;13:818-29.

20. Vincent JL, Moreno R, Takala J, Willatts S, De Mendonça A, Bruining H, et al. The SOFA (Sepsis-related Organ Failure Assessment) score to describe organ dysfunction/failure. Intensive Care Med. 1996;22:707-10.

21. National Kidney Foundation. K/DOQI clinical practice guidelines for chronic kidney disease: evaluation, classification, and stratification. Am J Kidney Dis. 2002:39(2 Suppl 1):S1-266.

22. Pickering JW, Endre ZH. Back-calculating baseline creatinine with MDRD misclassifies acute kidney injury in the intensive care unit. Clin J Am Soc Nephrol. 2010;5:1165-73.

23. Taylor Jr FB, Toh $\mathrm{CH}$, Hoots WK, Wada H, Levi M. Towards definition, clinical and laboratory criteria, and a scoring system for disseminated intravascular coagulation. Thromb Haemost. 2001;86:1327-30.

24. Hanley JA, McNeil BJ. The meaning and use of the area under a receiver operating characteristic (ROC) curve. Radiology. 1982;143:29-36.

25. Takano S, Kimura S, Ohdama S, Aoki N. Plasma thrombomodulin in health and diseases. Blood. 1990;76:2024-9.

26. Iba T, Yagi Y, Kidokoro A, Fukunaga M, Fukunaga T. Increased plasma levels of soluble thrombomodulin in patients with sepsis and organ failure. Surg Today. 1995;25:585-90.

27. Boldt J, Wollbrück T, Sonneborn S, Welters A, Hempelmann G. Thrombomodulin in intensive care patients. Intensive Care Med. 1995:21:645-50.

28. Itenov TS, Jensen JU, Ostrowski SR, Johansson PI, Lundgren JD, Bestle MH. Endothelial damage signals refractory acute kidney injury in critically ill patients. Shock. 2017:47:696-701.

29. Kazama M. Soluble thrombomodulin: a specific parameter of endothelia injury [in Japanese]. Rinsho Ketsueki. 1991;32:103-7.
30. Mishra J, Ma Q, Prada A, Mitsnefes M, Zahedi K, Yang J, et al. Identification of neutrophil gelatinase-associated lipocalin as a novel early urinary biomarker for ischemic renal injury. J Am Soc Nephrol. 2003;14:2534-43.

31. Han WK, Bailly V, Abichandani R, Thadhani R, Bonventre JV. Kidney injury molecule-1 (KIM-1): a novel biomarker for human renal proximal tubule injury. Kidney Int. 2002;62:237-44.

\section{Submit your next manuscript to BioMed Central and we will help you at every step:}

- We accept pre-submission inquiries

- Our selector tool helps you to find the most relevant journal

- We provide round the clock customer support

- Convenient online submission

- Thorough peer review

- Inclusion in PubMed and all major indexing services

- Maximum visibility for your research

Submit your manuscript at www.biomedcentral.com/submit

) Biomed Central 